\section{Naso-laryngeal distances correlate with body size and are not correctly reflected in commercially available preformed nasal tubes}

\author{
C. Massoth ${ }^{1}$, C. Schülke ${ }^{2}$, M. Dahrmann ${ }^{1}$, R. Weiss ${ }^{1}$, T. Prien ${ }^{1}$, M. Wenk ${ }^{1}$
}

${ }^{1}$ Department of Anesthesiology, Intensive Care and Pain Medicine, University Hospital of Muenster, Muenster (Germany) ${ }^{2}$ Department of Clinical Radiology, University Hospital Muenster - Muenster (Germany)

\section{Background}

Preformed nasal endotracheal tubes (NET) have a predefined insertion depth due to their curved design. While size indication refers to internal diameter, there is a considerable variability in the corresponding lengths of same sized tubes of different manufacturers, therefore choosing the best fitting NET is difficult.

\section{Objective}

-to describe nasolaryngeal distances (NLD) in an adult population using high resolution $\mathrm{CT}$ scans

-to compare these data to available NETs of several manufactures

\section{Methods}

Ethics Committee approval: 2017-073-f-S

Analysis of 388 CT scans of head, neck and upper thorax:

NLD was defined from the nares to the lower border of the thyroid cartilage and segmented based on anatomic landmarks (Figure 2)

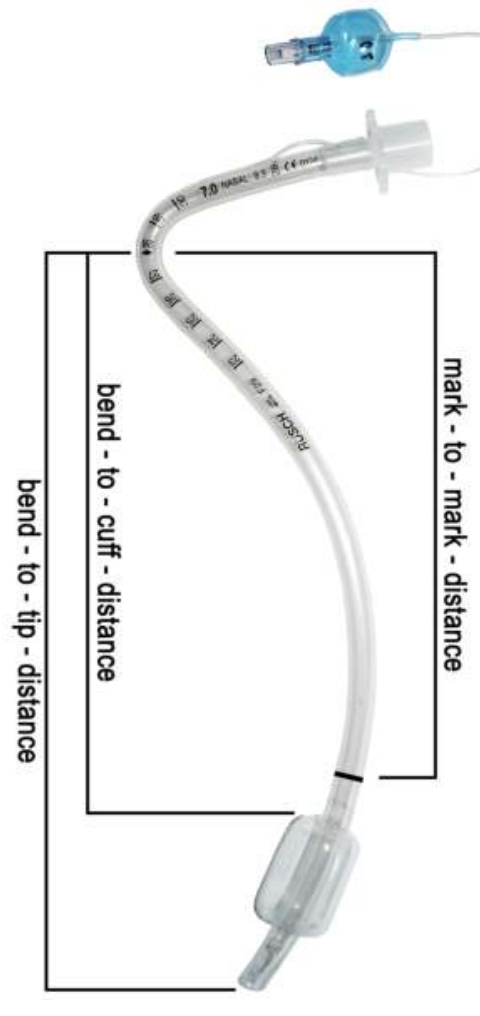

Figure 1

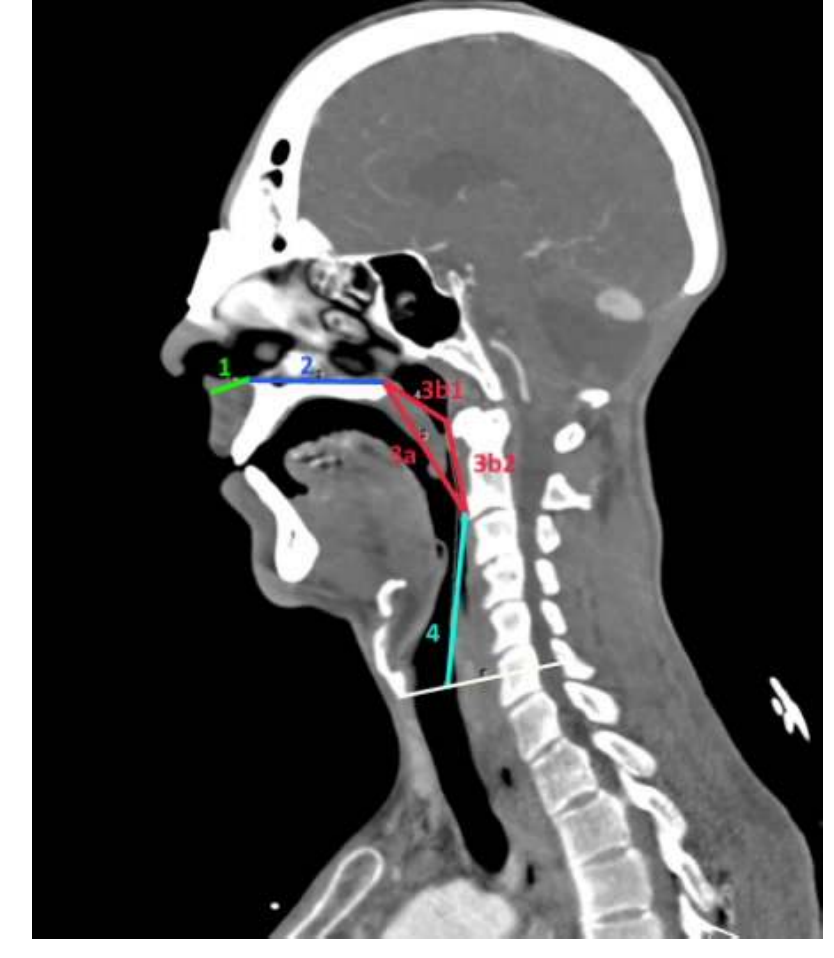

Figure 2:

NLDmean: $1+2+(3 a+3 b 1+3 b 2) / 2+4$
NETs from different manufacturers were measured (Figure 1).

A required bend-to-cuff-distance (RBTC) as most representable distance for the naso-laryngeal section was calculated, derived from CT measurements, then compared to acutal tube measurements

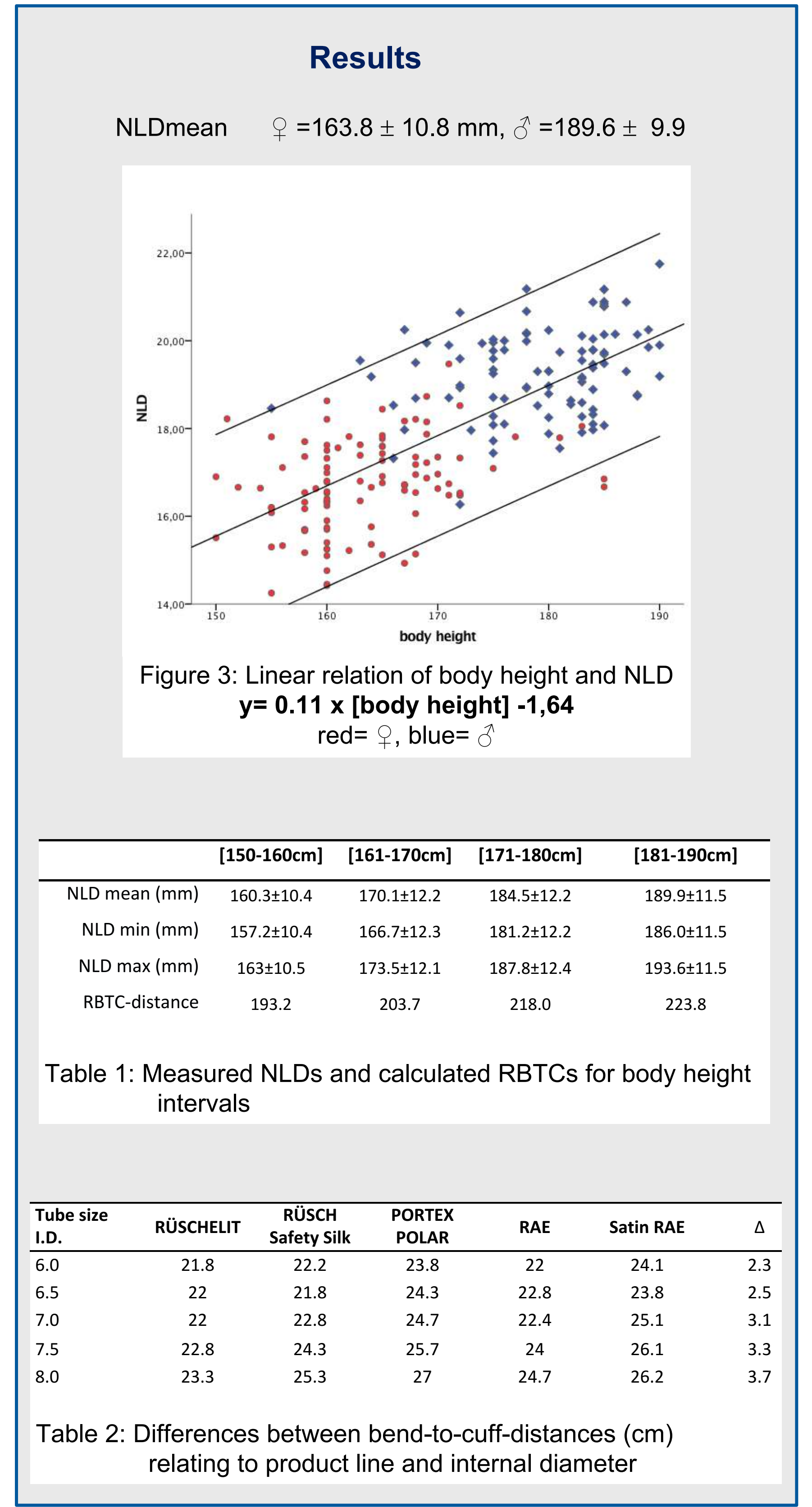

\section{Conclusion}

$\Rightarrow$ average naso-laryngeal distances can be correlated with body height

$\Rightarrow$ calculation of NLD is feasible, building a strong basis for choosing a tube with the required section length

$\Rightarrow$ manufacturers' tube lengths and intubation guide marks vary substantially

$\Rightarrow$ lengths do not always correlate with radiographic measurements, carrying the danger of cuff malposition 